\title{
Building vibrations induced by human activities: a benchmark of existing standards
}

\author{
Georges Kouroussis ${ }^{a}$, Calogero Conti and Olivier Verlinden
}

Service de Mécanique Rationnelle, Dynamique et Vibrations, Université de Mons, Faculté Polytechnique, Boulevard Dolez 31, 7000 Mons, Belgique

Received 7 January 2014, Accepted 15 May 2014

\begin{abstract}
Vibrations in buildings impact the behaviour of structures and humans, for sources defined as internal or external to the building. Besides the comparison of vibrations to fixed limits, the choice of a relevant indicator is unavoidable and this indicator is defined differently according to the selected reference text. Various standards (or directives similar to standards) exist and this paper focuses on the most important and the most used ones, for ground vibrations induced to buildings or for human exposure inside buildings. In addition to the interest of comparing values to well-defined and well-known limits, the knowledge of these standards allows the use of suitable indicators. Various vibration signals are used, from a simple harmonic signal to complex vibrations generated by railway traffic, in order to present a relevant analysis of severity of each norm. It turns out that criteria noticeably vary from one reference to another one, and that the thresholds are different for each standard.
\end{abstract}

Key words: Ground vibrations / standards / human effect / building

\section{Introduction}

In a growing number of situations, the influence of vibrations on structural damage in buildings and on people inside buildings can no longer be neglected. Among all the difficulties associated with the measurement of vibrations, the choice of a relevant indicator is most critical, and is often made by relying on standards, especially in the context of building design or diagnosis. This includes the impact of vibration on people inside buildings located in the vicinity of external sources of vibration. Figure 1 illustrates the transmission path of possible external sources, showing the complexity of soil/structure interaction and of effective means for vibration isolation. If various sources exist, they do not act in the same way, the generated level depends on the type of source (rail and road traffic, underground traffic, soil compaction, blasts...), the soil configuration (surface geometry, presence of a more or less rigid layer, water saturation of soils), the principal mode of propagation (body and surface waves), and obviously the distance from the source.

Unlike noise, vibrations are described by various indicators. Two problems are commonly examined, most often within a single study: the human perception and the

\footnotetext{
a Corresponding author:

georges.kouroussis@umons.ac . be
}

damages on buildings. In the case of people residing in buildings, they receive vibrations passively and this plays a role on health and comfort. Vibrations also affect the integrity of structures by imposing dynamic loads sufficient to cause structural fatigue (cracks are often the first visual impact of excessive stresses). The interest of engineers in problems of impact of vibrations on buildings is understandable. Consequently, they must evaluate the possible damage caused by their processes and ensure that the level of generated vibrations in buildings will not jeopardize the necessary comfort of people. To assist them, several standards exist, which define adequate procedures and assessments. The most important ones are:

- the German standards DIN 4150-2 [1] and DIN 4150-3 [2], formulated by the technical comity Arbeitsausschüß 00.50.00 and used in Germany, in Belgium and other European countries,

- the international standards ISO [3,4], which are often considered as a reference for comfort evaluation,

- the Swiss standards SN 640 312a [5] for the building damages only,

- the recommendations [6] of the United States Department of Transportation (USDT) on the assessment of potential vibration impacts resulting from high-speed train lines. 


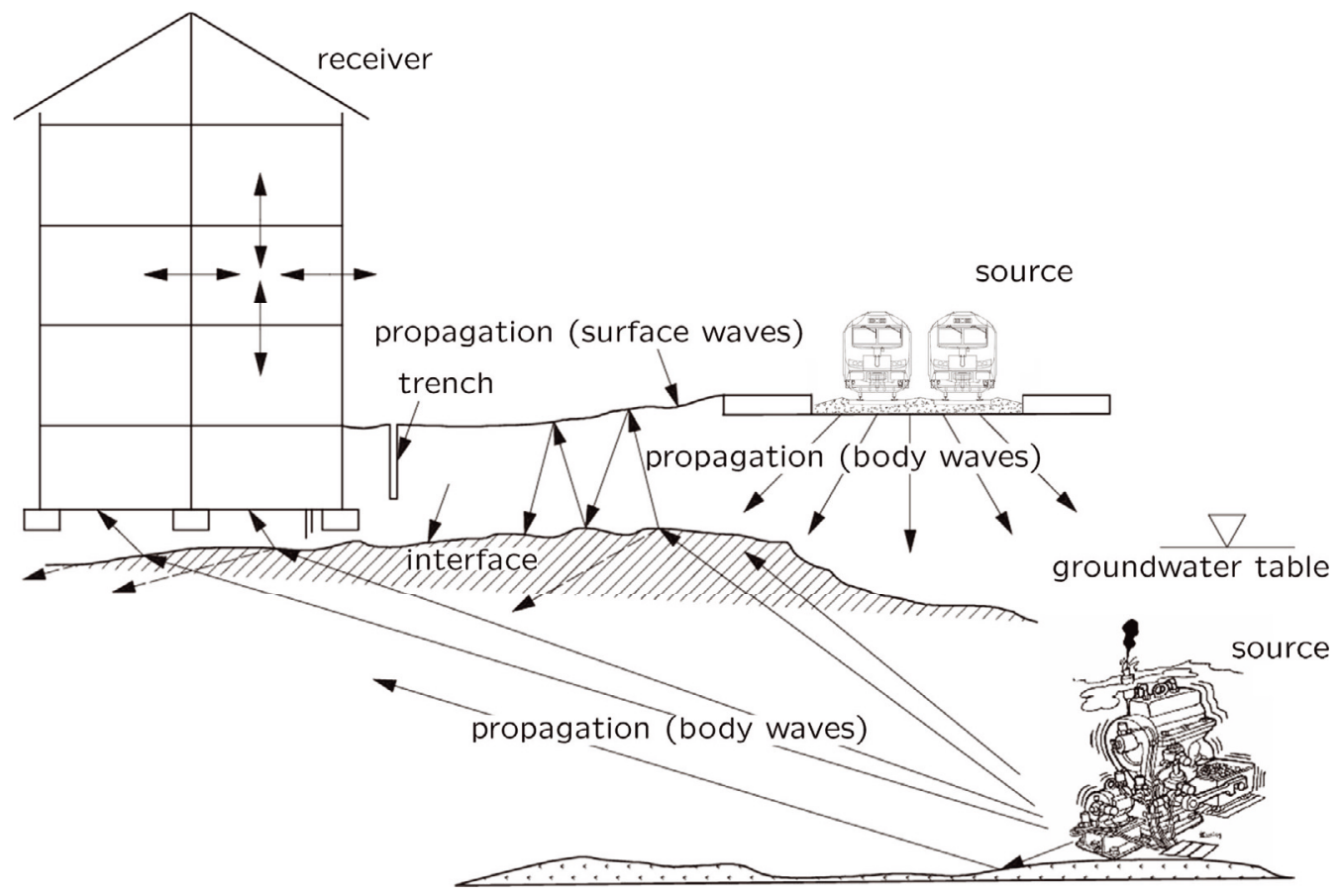

Fig. 1. Sketch illustrating the transmission of vibrations to buildings.

All these baselines represent the most used assessment guidelines for measurement and interpretational methodologies.

Research on recognizing the influence of vibrations and definition of criteria abounds on literature (see for example [7-9]). The perception of threshold for specific situations is often analysed. However, reflections about the retained primary vibration indicator are scarce, which is unfortunate, as when an indicator is retained, it masks important information. For example, an effective value gives an overview of the motion level but may hide shortterm and transient vibrations. Different methods are associated to these working documents and proposed evaluations are based on different indicators with, at first glance, any correlation.

The purpose of this paper is to compare the aforementioned guidelines, to analyse the associated criteria and to present the most interesting vibration indicators, based on the authors' experience. Our first step is to introduce the methodology. Two kinds of signal are then chosen for this study: simple harmonic motions and railway-induced ground vibrations. It is important, in discussing the obtained results, i.e. the effects of vibration on humans or on building, to define exactly the methodology of assessment of the influence of transport vibrations on people inside existing buildings and on buildings themself.

\section{Description of standard methodology}

This section presents the main standards and national recommendations related to ground-borne vibrations. For convenience, the reference frame and the associated basic axes illustrated in Figures 2 and 3 are adopted for human evaluation and structural evaluation, respectively.

The purpose is not to describe in detail the associated methods but to emphasize on their main particularities. All assume that the ground vibrations are usually in the range $1-80 \mathrm{~Hz}$, the higher frequencies having a small influence on ground motion amplitudes due to the high material damping of soils.

\subsection{ISO 2631 standards}

The International Organization for Standardization gives general recommendations about the evaluation of human exposure to whole-body vibrations. The first part of the standard [3] is dedicated to vibrations felt inside vehicles but the second part [4] gives information about vibrations in buildings and their transfer to persons. In 1997, the evaluation procedure was changed with the definition of frequency-dependent filters related to the activity, the human position (standing, sitting or sleeping) and the direction of vibration. A weighted acceleration $\mathrm{a}_{w}$ is derived from the time history of the measured acceleration $^{1}$. A root-mean squared $(r m s)$ value is then calculated and describes the smoothed vibration amplitude by supposing that the human body responds to an average

\footnotetext{
1 The old version of ISO 2631-1 (1985 version) was based on a comparison of the frequency signal to a third-octave band limit curve. The various guidelines for comfort and health were simply defined by a multiplication factor. The last version represents a radical change.
} 


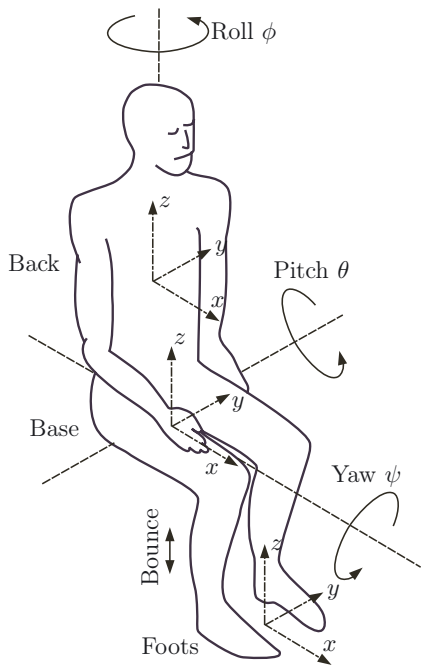

(a) Seated position

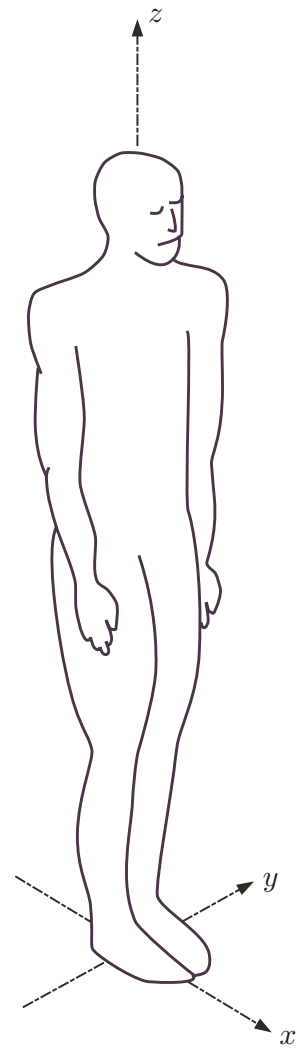

(b) Standing position

Fig. 2. Definition of axis for human body (adapted from [3]).

vibration amplitude during a recorded time $0 \leqslant t \leqslant T$ :

$$
\mathrm{a}_{w}=\sqrt{\frac{1}{T} \int_{0}^{T} a_{w}^{2}(t) d t}
$$

A guide on the effect of vibrations on comfort and perception is given with valuable limits defining grades of various magnitudes of reaction to vibrations. The effects on health is however less well described; only two bounds are given (probable risk if above the upper limit, improbable risk below the lower limit) without any further explanation for the case when the calculated value lies within the intermediate region. The time duration of vibration only plays a role for health evaluations.

In the case of evaluations inside buildings [4], it is noteworthy that, one single filter is defined, independent of the measurement direction and human position, focusing on the frequency range $1-20 \mathrm{~Hz}$ (Fig. 4).

\subsection{DIN 4150 standards}

Because a vibration is often non-stationary, the DIN 4150-2 standard [1] proposes the use of a running rootmean square applied to the velocity signal. A weighted time-averaged signal is thus defined by:

$$
K B_{F}(t)=\sqrt{\frac{1}{\tau} \int_{0}^{T} K B^{2}(\xi) e^{-\frac{t-\xi}{\tau}} d \xi}
$$

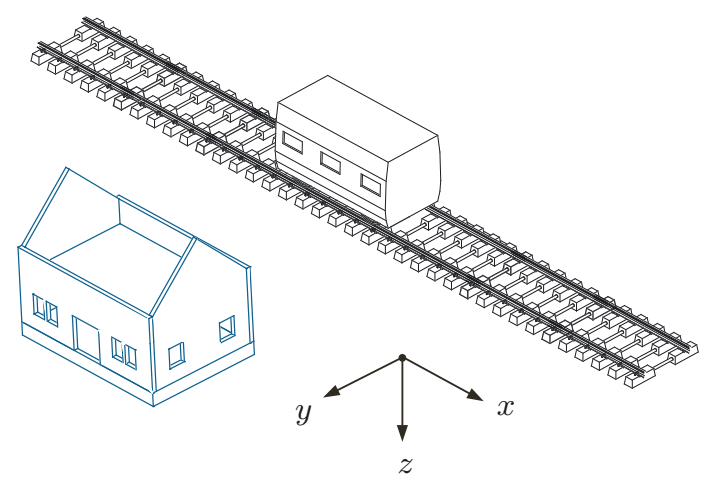

Fig. 3. Definition of axis for building vibration analysis.

where the weighted velocity signal $K B(t)$ is obtained by flowing the original velocity signal through the high-pass filter

$$
H_{K B}(f)=\frac{1}{\sqrt{1+\left(\frac{5.6}{f}\right)^{2}}}
$$

The filter is a function of the frequency $f$. The integration time $\tau$ to run the averaging is equal to $0.125 \mathrm{~s}$, which allows taking into account transient phenomena like impacts or shocks that would otherwise be masked if a simple rms operation was performed. Although no unit is specified in the standards, the associated unit is clearly 


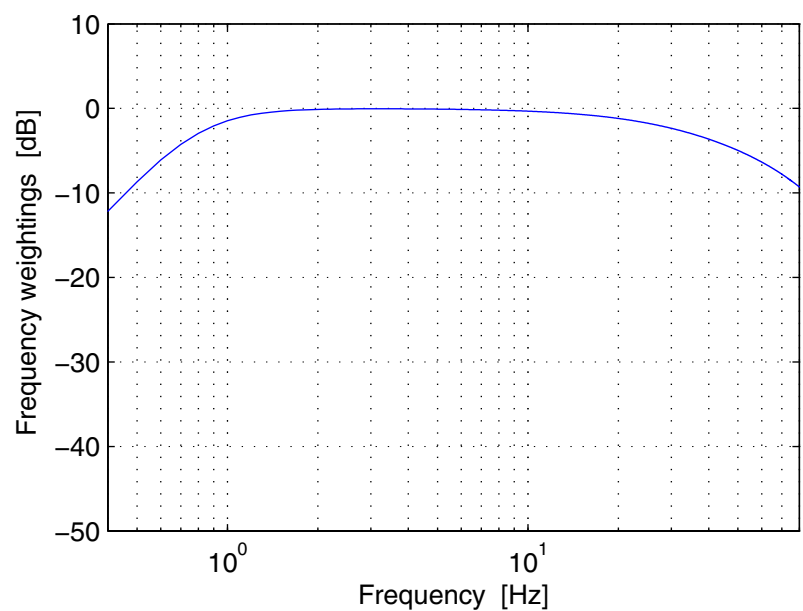

Fig. 4. Frequency weighting curve for human exposure inside buildings.

$\mathrm{m} / \mathrm{s}$ (or more usually $\mathrm{mm} / \mathrm{s}$ ). Only comfort is then assessed by comparing the maximum level $K B_{F, \max }$ with three guideline limits denoted $A_{u}, A_{o}$ and $A_{r}$, for a whole evaluation and for the short-term frequency vibrations as well as.

DIN 4150-3 standard [2] is solely dedicated to the effect on structure. The peak particle velocity $P P V$, defined as the maximum absolute amplitude of the velocity time signal, is calculated and compared to limits depending on the dominant signal frequency. If multiple directions are measured, the maximum of the three components is retained. By taking into account the velocity as primary indicator, it is possible from one signal to evaluate both human comfort and building damage.

\subsection{USDT recommendations}

Considering that vibrations consist of rapidly fluctuating motions, a decibel scale was adopted by the U.S. Department of Transportation in order to evaluate the vibration impact of passing high-speed train [6]. As for the description of noise, this scale serves to compress the range of numbers required to describe the vibration velocity level, and is defined as:

$$
V_{d B}=20 \log _{10} \frac{v_{r m s}}{5 \times 10^{-8}}
$$

where $v_{r m s}$ is the root mean square amplitude of the velocity time history. Notice that no weighting is applied to the signal, on the contrary to ISO standards. An equivalent standardized weighted vibration level $V L_{d B}$ has been used in Japan to evaluate human response to vibration: for frequencies greater than $8 \mathrm{~Hz}$, the following relationship exists [6]

$$
V L_{d B}=V_{d B}-21
$$

Typical levels of ground-borne vibrations are also provided in reference [6].

\subsection{SN 640 312a standard}

The Swiss standard [5] is similar to its German counterpart DIN 4150-3 since it also uses a $P P V$, defined as the norm of the vector velocity

$$
P P V=\sqrt{v_{x}^{2}+v_{y}^{2}+v_{z}^{2}}
$$

If one direction is dominant in terms of amplitude, both definitions are equivalent. The guidelines are also different, with the introduction of an excitation frequency (occasional, frequent or continuous excitation). Limits are a function of the dominant signal frequency.

\section{Comparison using harmonic signals}

A harmonic signal is certainly the simplest vibration record. It can be encountered in practice when the vibration is dominated by an important resonance mode and/or when the excitation is clearly mono-frequency. To be concise, the vibration amplitude is imposed so as to define a vibratory motion by

$$
v(t)=A \sin (2 \pi f t)
$$

where the amplitude $A$ is constant and the frequency $f$ can vary from 1 to $100 \mathrm{~Hz}$.

A first analysis evaluates human exposure. In a first step, the trends of $K B_{F}(t)$ are previewed, since this indicator uses "non-usual" operations compared to the other guidelines. Figure 5 presents this indicator as a function of the frequency $f$, showing its time history (Fig. 5a) as well as its maximum value (vibratory dose - Fig. 5b). The level clearly tends to the effective value of $0.707 A$. The relationship between amplitude $A$ (which represents the $P P V$ ) and $K B_{F, \text { max }}$ can be established as follow

$$
K B_{F, \max }=\frac{\sqrt{2}}{2} \frac{P P V}{\sqrt{1+(5.6 / f)^{2}}}
$$

for sufficient values of frequency $f$. Otherwise, a multiplying coefficient can be applied (for example 0.9 for $f=10 \mathrm{~Hz}$ ). If the acceleration is taken into account (for example, by the ISO weighted acceleration), equation (8) can be rewritten as

$$
\begin{aligned}
K B_{F, \max }= & \frac{1000}{2 \pi f} \frac{\mathrm{a}_{w}}{\sqrt{1+(5.6 / f)^{2}}} \\
& \times\left(-0.0056 f^{2}+0.11 f+0.071\right)
\end{aligned}
$$

Equation (9) is obtained by quadratic curve-fitting and is valid in the frequency range 2 to $10 \mathrm{~Hz}$ where the ISO weighted filter amplitude is equal to 1 . Also, notice that the unit of acceleration in equation (9) is $\mathrm{m} / \mathrm{s}^{2}$. In the frequency range $10-20 \mathrm{~Hz}$, a new fitting provides the formula

$$
\begin{aligned}
K B_{F, \max }= & \frac{1000}{2 \pi f} \frac{\mathrm{a}_{w}}{\sqrt{1+(5.6 / f)^{2}}} \\
& \times\left(-0.0005 f^{2}+0.02 f+0.48\right)
\end{aligned}
$$




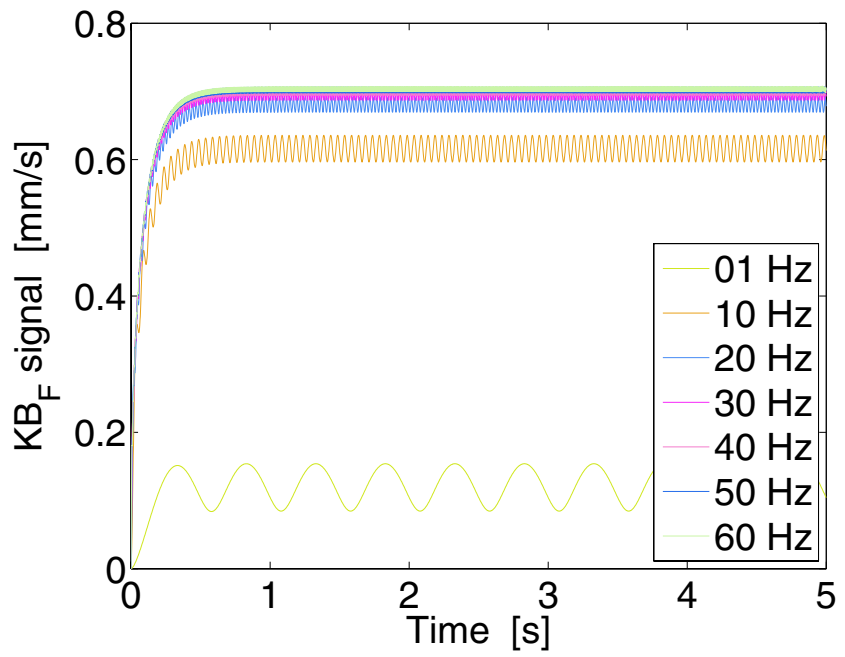

(a) Weighted time history $K B_{F}(t)$

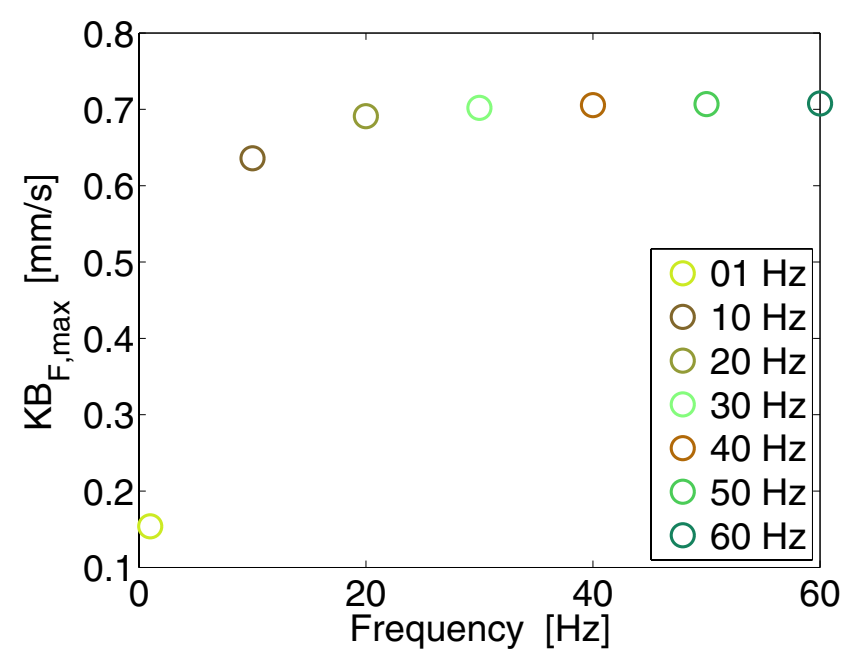

(b) Vibratory dose $K B_{F, \max }$

Fig. 5. DIN 4150-2 standards putting into practice on harmonic signals.

Simpler methods (as those proposed by the older versions of the DIN 4150-2 standard) propose a constant corrective factor in place of the fitting term, according to the type of excitation and activity.

Full iso- $K B_{F, \max }$ curves, for both harmonic velocities and accelerations, can be deduced from these formulas, as illustrated in Figure 6. These graphs give a simple overview of the equivalence between the approach proposed by the German guidelines and the ISO and American ones for the human exposure.

Including the limits proposed by these baselines allows the comparison in terms of comfort evaluation. Figure 7 shows the results with a harmonic signal of amplitude $A=5 \mathrm{~mm} / \mathrm{s}$, sufficiently high to exceed the proposed limits, and an increasing signal frequency (note that an increase of amplitude translates the calculated curves vertically). Several comments can be made on the results. The limit is overstepped at $8 \mathrm{~Hz}$, according to DIN, and at $15 \mathrm{~Hz}$ according to ISO. Notice that the definitions are different: for the DIN standards, limit $A_{o}$ represents the borderline case from which the annoyance is confirmed for any event. $A_{u}$ represents the limit below which the annoyance is not detected. The number of events only plays a role if the vibration level is comprised between $A_{u}$ and $A_{o}$ (the supplementary limit $A_{r}$ is used in this case). In a different spirit, the ISO standard defines various grades of annoyance. This means that a harmonic vibration signal can be assumed strong for the DIN standard and low for the ISO one if the frequency lies between 8 and $15 \mathrm{~Hz}$ !

The second analysis is the effect on buildings. Only the German and Swiss standards give assessment methods, correlating the $P P V$ to the structural stress. Figure 8 displays the associated limits, showing that they are close to each other. The Swiss standard presents the undeniable advantage to consider explicitly the frequency of events. It is also observable that the DIN limits are approximately to the SN limits for frequent to continuous excitations.

\section{Comparison with complex signals: the case of railway-induced ground vibrations}

Problems of railway-induced ground vibrations have received considerable attention in the last few years. Due to the development of new networks, complaints are more and more frequent to such an extent as to hinder the prosperity of this solution to traffic congestion. Scientifically speaking, the mastering of ground vibration sources and their effects on neighbouring structures are insufficiently known due to the difficulty to quantify all the components in play in the generation and the propagation of ground waves. To propose relevant and sufficient results, a numerical model was developed by Kouroussis et al. [10] and validated in several cases. This prediction scheme is based on a two-step approach, separating the vehicle/track and the soil dynamics calculations, in order to focus on detailed models of vehicles.

Figure 9 shows results from such a numerical model which describes the passage of a tram on a singular rail surface defect. It presents some time histories of the vertical velocity at the ground surface at various distances from the track and for a vehicle speed of $30 \mathrm{~km} / \mathrm{h}$. The corresponding frequency spectra are also added, showing that the main response frequency is around $20 \mathrm{~Hz}$ (a mean of $21.1 \mathrm{~Hz}$ is calculated for all the distance between 2 and $20 \mathrm{~m}$ ). These results are typical of important wheel/rail interactions where the vehicle dynamics is clearly visible in the ground-borne vibrations [11].

Figure 10 shows the corresponding indicators based on the aforementioned guidelines. The three directions are analysed. While the vibration level in the vertical direction is the greatest, the horizontal vibrations cannot be ignored. This statement confirms the good practice rules observed in experimental assessments to always record the three directional components of vibratory nuisances. The four indicators, namely the peak particle velocity, the maximum weighted acceleration, the vibration 


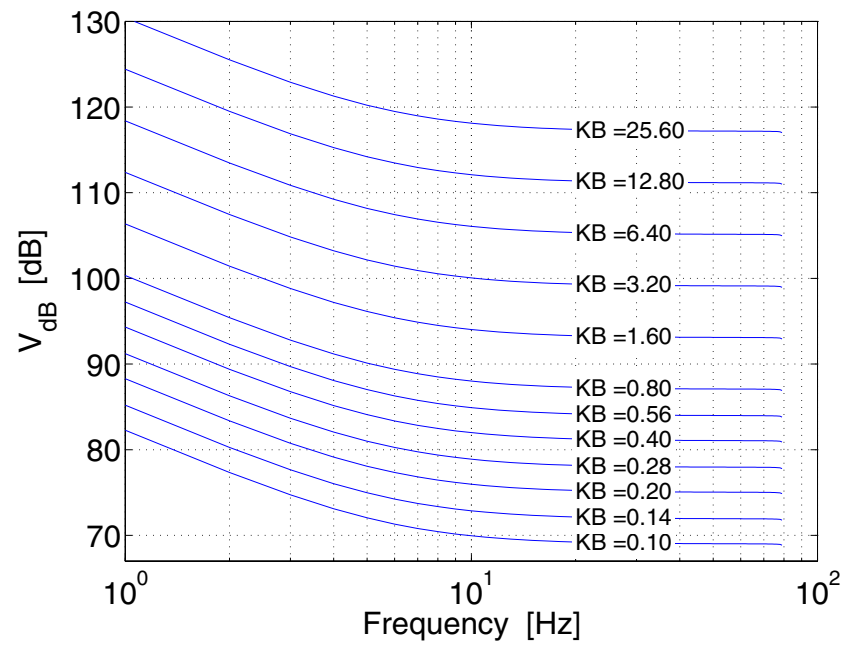

(a) For harmonic velocities

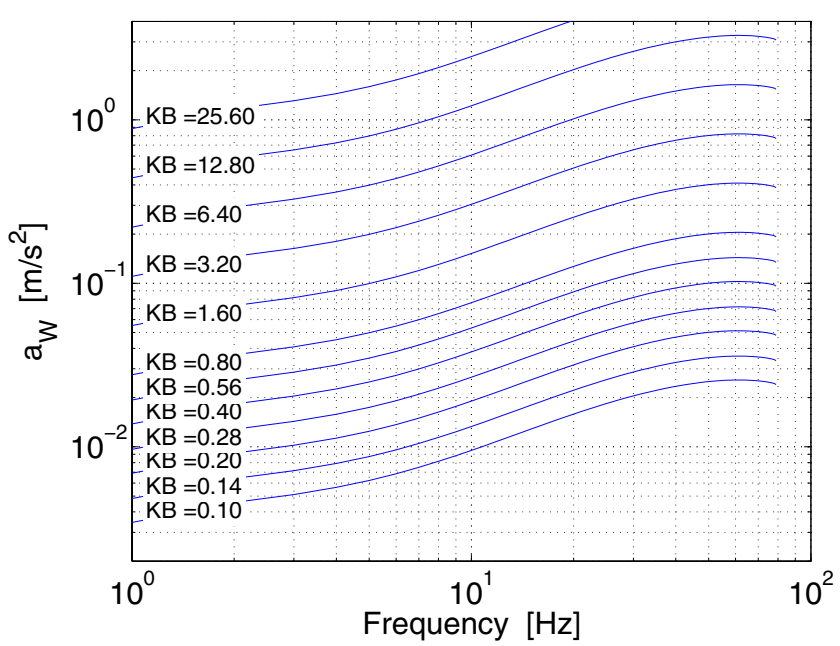

(b) For harmonic accelerations

Fig. 6. Iso- $K B_{F, \max }$ curves.

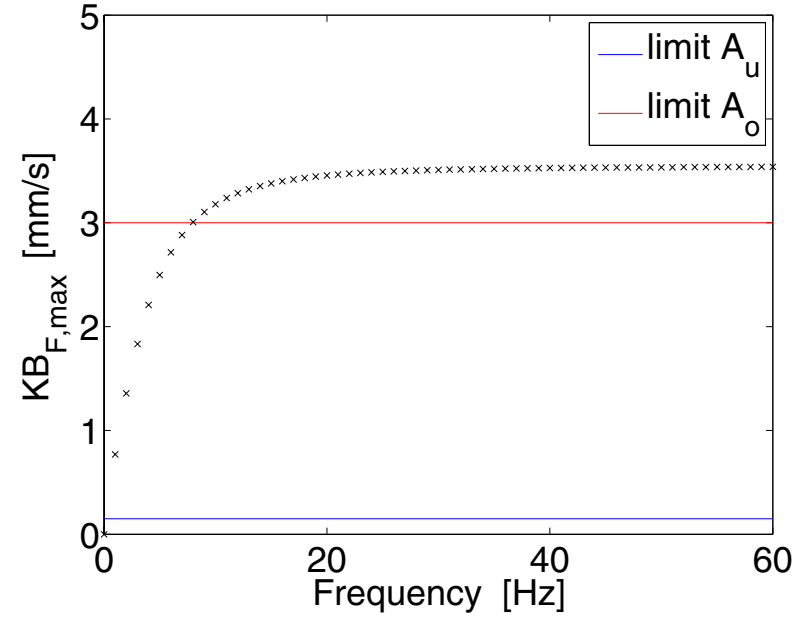

(a) Assessment according to the DIN 4150-2

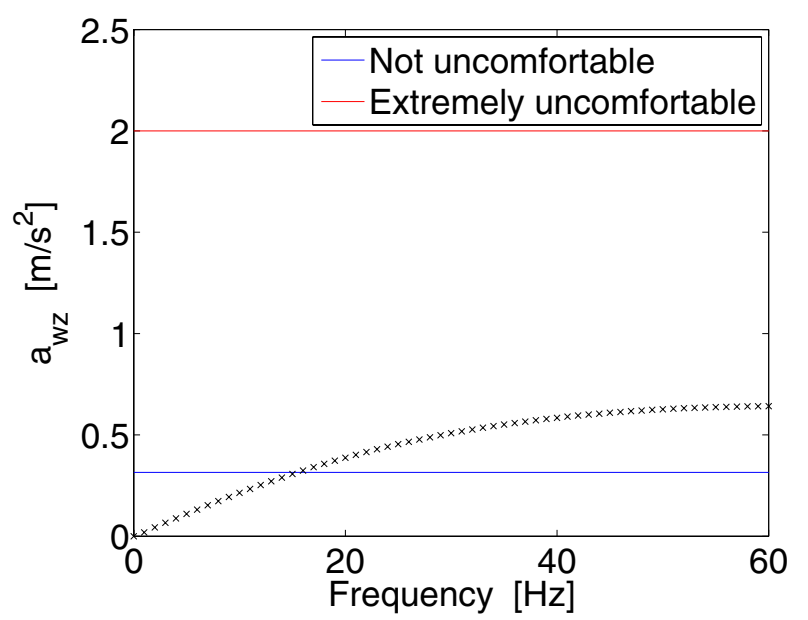

(b) Assessment according to the ISO 2631-2

Fig. 7. Comparison between standards DIN 4150-2 and ISO2631-2 for harmonic signals.

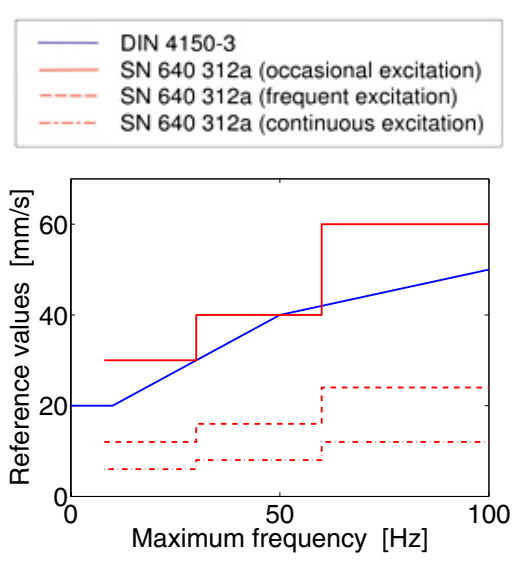

(a) Industrial buildings

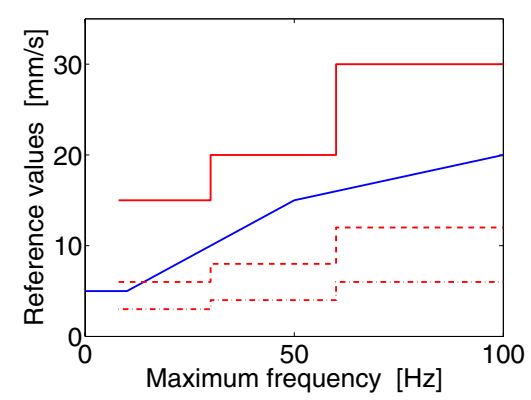

(b) Standard buildings

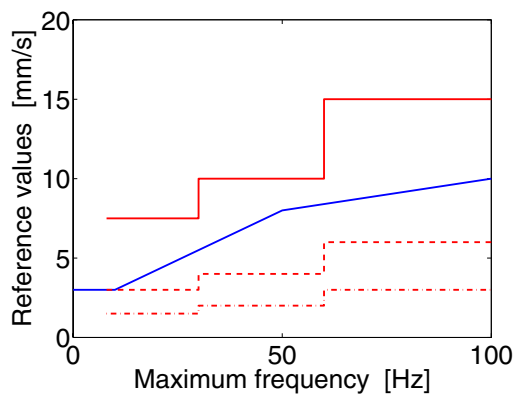

(c) Sensitive buildings

Fig. 8. Comparison between DIN 4150-2 and SN640 312a for the effects on building according to the type of structure. 

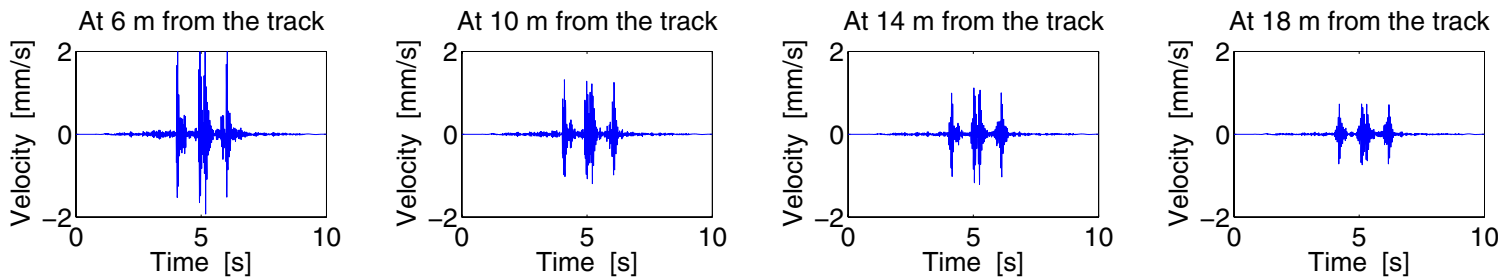

(a) Time histories
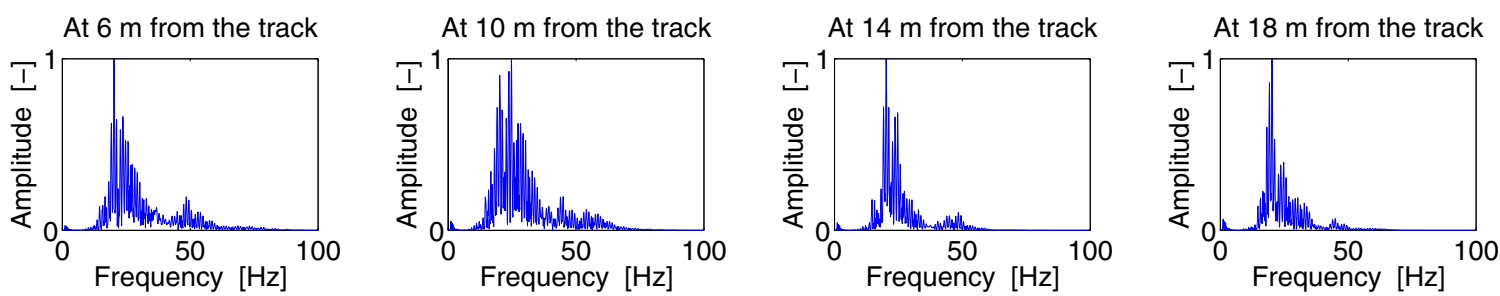

(b) Frequency contents

Fig. 9. Predicted results for vertical ground velocities due to the passage of a tram over a singular rail surface defect.

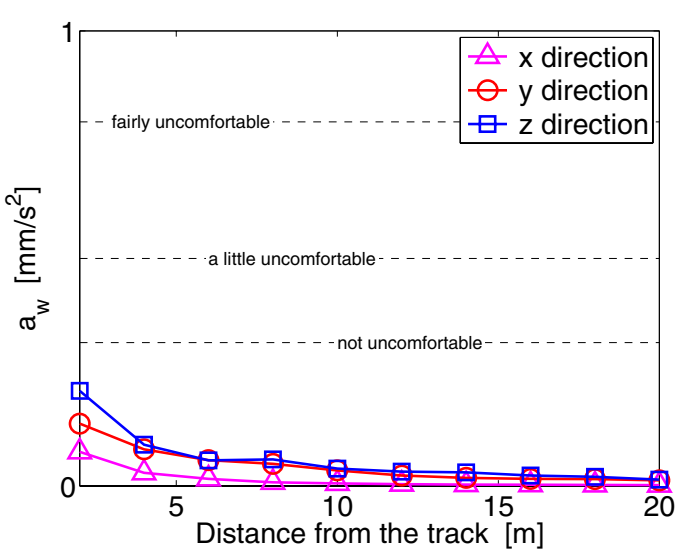

(a) Maximum weighted acceleration

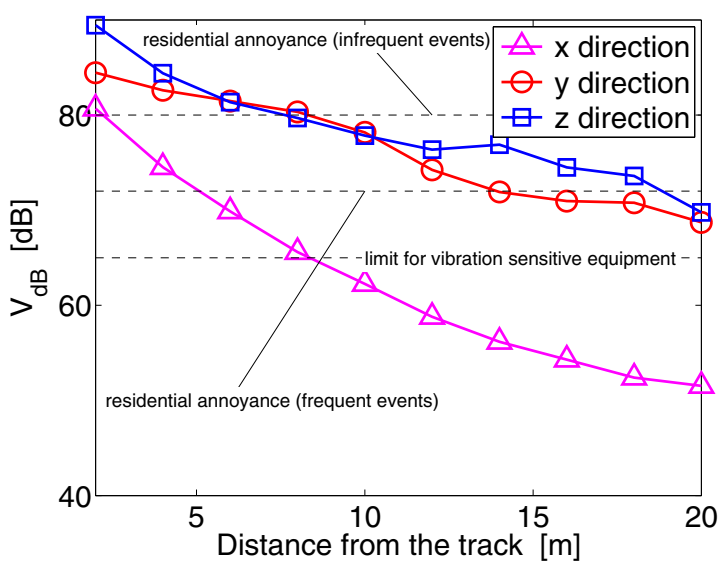

(c) Vibration velocity level

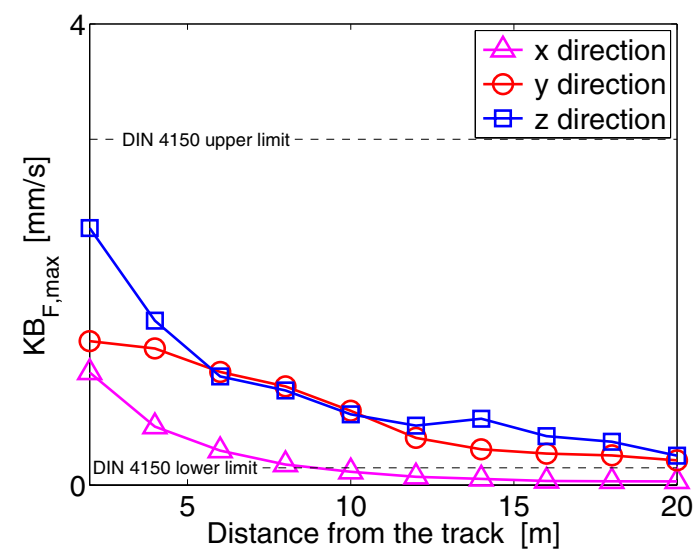

(b) Maximum weighted velocity dose

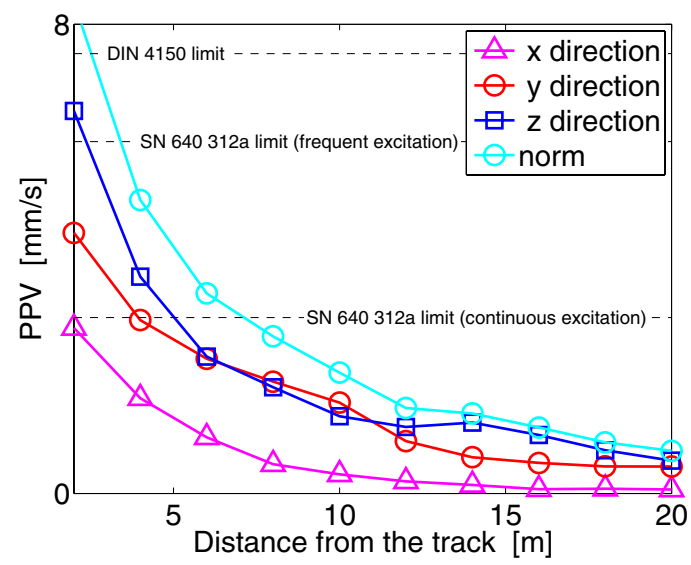

(d) Peak particle velocity

Fig. 10. Calculated indicators associated to the passage of a tram over a singular rail surface defect and as a function of the distance from the track. 
Table 1. Standard analysis summary for ground-borne vibration assessment.

\begin{tabular}{|c|c|c|c|}
\hline Standard & Parameter & Limits & Conditions of use \\
\hline \multicolumn{4}{|c|}{ Effect on structures } \\
\hline \multirow{2}{*}{ DIN 4150-3 } & peak particle velocity & $3 \mathrm{~mm} / \mathrm{s}$ & (very) sensitive building \\
\hline & (the higher component) & $5 \mathrm{~mm} / \mathrm{s}$ & dwelling place \\
\hline \multirow{2}{*}{ SN $640312 \mathrm{a}$} & peak particle velocity & $3 \mathrm{~mm} / \mathrm{s}$ & frequently excited sensitive building \\
\hline & (the higher component) & $6 \mathrm{~mm} / \mathrm{s}$ & normally excited building \\
\hline \multicolumn{4}{|c|}{ Effect on persons in building } \\
\hline \multirow{2}{*}{ ISO $2631-2$} & weighted $r m s$ & $0.315 \mathrm{~m} / \mathrm{s}^{2}$ & not or a little uncomfortable \\
\hline & acceleration & $2 \mathrm{~m} / \mathrm{s}^{2}$ & extremely uncomfortable \\
\hline \multirow{4}{*}{ USDT } & \multirow{4}{*}{ rms velocity } & \multirow{2}{*}{$0.10 \mathrm{~mm} / \mathrm{s}$} & residential area \\
\hline & & & frequent ( $>70$ passbys per day) \\
\hline & & \multirow{2}{*}{$0.26 \mathrm{~mm} / \mathrm{s}$} & residential aera \\
\hline & & & infrequent ( $<70$ passbys per day) \\
\hline \multirow{2}{*}{ DIN 4150-2 } & weighted dose & $0.15 \mathrm{~mm} / \mathrm{s}$ & residential area \\
\hline & $K B_{F, \max }$ & $0.10 \mathrm{~mm} / \mathrm{s}$ & sentitive area \\
\hline
\end{tabular}

velocity level and the maximum weighted velocity dose, present the same tendency: a strong decrease in level in the near field (up to $5 \mathrm{~m}$ ) and a weak reduction above $10 \mathrm{~m}$ from which the $y$ and $z$ direction amplitudes tend to the same values. For the $P P V$ graph, the norm of the velocity vector is calculated to facilitate the comparison with the SN 640 312a standard.

Adding the guideline limits to each plot reveals different observations for human exposure:

- For the ISO 2631 standard, vibratory nuisance is avoided for any distance from the track.

- For the DIN 4150, no direct conclusion can be drawn. The number of events must be taken into account.

- The recommendation of USDT clearly shows that infrequent events are tolerated for vibrations at distances beyond $8 \mathrm{~m}$.

Regarding the effects on buildings, the observations are also different depending on which recommendation is considered. For DIN 4150, structural damages cannot appear at any distance from the track. For the SN640 312a standard, the worst case appears at distances smaller than $7 \mathrm{~m}$ and only for continuous vibrations.

\section{Conclusion}

Common standards for the evaluation of vibration annoyance which were reviewed in this work are summarized in Table 1. This paper also presented practical results based on mono-frequency excitation and railway-induced ground vibrations. It appears that the assessment problem is complex, since contradictory recommendations can be provided by the guidelines, both for human exposition and for the effects on structures. Additional research is called upon to provide a definitive assessment of the effects of vibrations.

\section{References}

[1] Deutsches Institut für Normung, DIN 4150-2: Structural vibrations - Part 2: Human exposure to vibration in buildings, 1999

[2] Deutsches Institut für Normung, DIN 4150-3: Structural vibrations - Part 3: Effects of vibration on structures, 1999

[3] International Organization for Standardization, ISO 2631-1: Mechanical vibration and shock - Evaluation of human exposure to whole-body vibration - Part 1: General requirements, 1997

[4] International Organization for Standardization, ISO 2631-2: Mechanical vibration and shock - Evaluation of human exposure to whole-body vibration - Part 2: Vibration in buildings $(1 \mathrm{~Hz}$ to $80 \mathrm{~Hz}), 2003$

[5] Schweizerische Normen-Vereinigung, SN-640312a: Les ébranlements - Effet des ébranlements sur les constructions (Swiss Standard on vibration effects on buildings), 1992

[6] U.S. Department of Transportation (Federal Railroad Administration), High-speed ground transportation. Noise and vibration impact assessment, Technical Report 293630-1, Office of Railroad Development Washington, 1998 
[7] J. Kawecki, K. Stypula, Methods of Assessment for Vibration Impact on People in Buildings, In: B.H.V. Topping ed., Proceedings of the Eleventh International Conference on Computational Structures Technology, Civil-Comp Press, Dubrovnik, Croatia, 2012

[8] K. Vogiatzis, Protection of the cultural heritage from underground metro vibration and ground-borne noise in Athens centre: The case of the Kerameikos archaeological museum and Gazi cultural centre, Int. J. Acoust. Vib. 17 (2012) 59-72
[9] M.G.R. Toward, M.J. Griffin, The transmission of vertical vibration through seats: Influence of the characteristics of the human body, J. Sound Vib. 330 (2011) 6526-6543

[10] G. Kouroussis, O. Verlinden, C. Conti, A two-step time simulation of ground vibrations induced by the railway traffic, J. Mech. Eng. Sci. 226 (2012) 454-472

[11] G. Kouroussis, O. Verlinden, C. Conti, Efficiency of resilient wheels on the alleviation of railway ground vibrations, Proc. IMechE, Part F: Journal of Rail and Rapid Transit 226 (2012) 381-396 